\section{Comprendiendo la experiencia y las necesidades al ser cuidador primario de un familiar con enfermedad de Alzheimer. Estudio de caso}

\section{Understanding the experience and needs to be the primary caregiver of a family member with Alzheimer's disease. Case study}

Miriam Rubio Acuña

Enfermera con diploma académico en el adulto mayor. Pontificia Universidad Católica de Chile. Profesor asistente adjunto. Escuela de Enfermería. Pontificia Universidad Católica de Chile.

\section{Correspondencia:}

Av. Vicuña Mackenna 4860. San Joaquín. Santiago de Chile.

E-mail:merubioa@uc.cl

\section{RESUMEN}

La enfermedad de Alzheimer (EA) afecta al 8\% de la población mayor de 65 ańos y al 30\% de la población mayor de 80 ańos, con un elevado

costo económico, social y humano. Propósito: aportar conocimiento comprensivo sobre la experiencia de ser cuidador de un familiar con EA.

Metodología: estudio de caso cualitativo de aproximación fenomenológica. Se realizó una entrevista en profundidad a una cuidadora de un familiar con EA. Para el análisis de la información se utilizó el método de Colaizzi.

Resultados: se revelaron las siguientes unidades de significado: experiencia fuerte debido al vínculo afectivo, sentirse violentada y aparición de sentimientos contradictorios, sensación de pérdida del ser querido, difícil decisión de institucionalización, búsqueda del sentido de

la enfermedad, y sentirse sobrepasada por la situación.

Conclusión: este estudio de caso pretende contribuir al conocimiento comprensivo de esta experiencia de cuidado.

PALABRAS CLAVE: enfermedad de Alzheimer, cuidador primario, estudio de caso.

\section{ABSTRACT}

Alzheimer's disease (AD) affects $8 \%$ of the population aged 65 years and $30 \%$ of the population over 80 years, with a high economic, social and human cost. Purpose: To provide comprehensive knowledge about the experience of being a caregiver for a family member with AD.

Methodology: qualitative study of phenomenological approach. Were interviewed in depth to a caregiver of a relative with $\mathrm{AD}$. For the data analysis method was used Colaizzi.

Results: We unveiled the following units of meaning: strong experience due to bonding, feeling violated and emergence of contradictory feelings, sense of loss of a loved one, difficult decision to institutionalization, search for meaning beyond the disease and feel about the situation.

Conclusion: This case study aims to provide comprehensive knowledge of the care experience.

KEYWORDS: Alzheimer's disease, primary caregiver, case study.

\section{- INTRODUCCIÓN}

Los avances tecnológicos y las mejores condiciones sociosanitarias han provocado un aumento de la esperanza de vida en Chile (78 años para la población general). Esta prolongación de la vida incide en una transición epidemiológica en la que nuevas patologías pasan a ocupar el foco de interés. Entre estas patologías, los trastornos demenciales ocupan un lugar de primacía, y no solo afectan a la persona que los presenta, sino que impactan también en la familia, el estado y la sociedad ${ }^{1}$.

La enfermedad de Alzheimer (EA) es un tipo de demencia caracterizada por una afectación precoz de la memoria seguida por un deterioro cognitivo de otras funciones superiores ${ }^{2}$. Afecta aproximadamente al $8 \%$ de la población mayor de 65 años y al 30\% de la población mayor de 80 años en los países con una alta expectativa de vida, con un elevado costo económico, social y fundamentalmente humano ${ }^{3}$.

El deterioro cognitivo en la demencia puede acompañarse de alteraciones en otras áreas de la personalidad y asociarse a ansiedad, depresión y trastornos del sueńo. Con frecuencia, los individuos se muestran agresivos y expresan ideas de perjuicio, que la mayoría de las veces adquieren un carácter propio de delirio. Las más frecuentes son las que implican temas de persecución, por ejemplo el sujeto puede llegar a pensar que los demás le van a quitar las pertenencias a las que se encuentra aferrado (dinero, vivienda, joyas, entre otros) o bien puede creer que los objetos que no encuentra le han sido robados ${ }^{1}$; todo esto genera estrés en los cuidadores.

Así también, situaciones generadoras de malestar en el cuidador son la pérdida del ser amado, incapacidad para comunicarse con el paciente 
como lo hacía en el pasado, frustración, ansiedad e impotencia ante un problema que no puede resolver el cuidador ${ }^{4}$.

Por lo anteriormente mencionado, esta enfermedad es particularmente devastadora, no solo por el deterioro progresivo de la persona que la presenta, sino también por el impacto en la familia. Dentro de esta, la prestación de cuidados no acostumbra a repartirse por igual entre sus miembros, sino que existe un "cuidador principal o primario" sobre el que recae la mayor responsabilidad del cuidado. Por consiguiente, el cuidador es aquella persona que de forma cotidiana se hace cargo de satisfacer las necesidades básicas y psicosociales del enfermo o lo supervisa en su domicilio en su vida diaria ${ }^{5}$. Entre algunas de las características de este cuidador se encuentran ser mujer, familiar cercano (esposa o hija) y ama de casa ${ }^{2,6-8}$.

El cuidado constante de una persona enferma genera consecuencias en el cuidador, denominadas "carga", la cual puede ser objetiva o subjetiva. La primera se refiere a la alteración de la vida social del cuidador, la cantidad de tiempo y de dinero invertido en los cuidados del enfermo. La sobrecarga subjetiva es la percepción que tiene el cuidador de la repercusión emocional de las demandas o problemas relacionados con el acto de cuidar. Este último tipo de sobrecarga tiene mayor incidencia sobre la vida del paciente y sus familiares ${ }^{5}$.

A pesar de que el tema se encuentra ya estudiado, este se ha realizado principalmente desde un enfoque cuantitativo. Sin embargo, existen estudios cualitativos que abordan el tema, pero son bastantes escasos y además están desarrollados en otros contextos socioculturales sin considerar la perspectiva de los protagonistas que portan el fenómeno en estudio, por lo que no se conoce en profundidad lo que las personas sienten, viven y necesitan como cuidador primario de familiares con EA.

El propósito de este estudio es aportar conocimiento comprensivo en relación con la experiencia y las necesidades al ser cuidador primario de un familiar con EA, para posteriormente diseñar intervenciones adecuadas para estos cuidadores primarios.

\section{- METODOLOGÍA}

Estudio de caso cualitativo, de aproximación fenomenológica, ya que permite conocer el fenómeno tal y como se presenta, es decir desde el punto de vista de la experiencia de la realidad de estas personas, desde su mundo propio.

La fenomenología es tanto una filosofía como un método de investi-

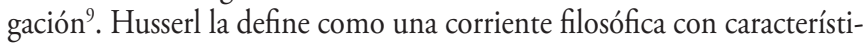
cas de trascendental, que constituye el método que permite describir el sentido de las cosas viviéndolas como fenómenos de conciencia.

El propósito de la investigación fenomenológica es describir las experiencias tal y como son vividas ${ }^{9}$, lo que permite conocer la experiencia humana en relación con un fenómeno de interés, desde la perspectiva de la persona que la vive. Por lo tanto, la realidad se considera subjetiva, ya que se encuentra influida por un marco de referencia propio ${ }^{10}$.

El participante es una persona que está viviendo la experiencia de ser cuidador primario de un familiar con Alzheimer durante más de 6 meses.

Para la recolección de la información se utilizó la técnica de entrevista individual en profundidad, la cual permite conocer el fenómeno tal y como se presenta, lo que posibilita revelar la experiencia. El objetivo de esta técnica es obtener una idea profunda de la experiencia de los participantes ${ }^{9}$. Este tipo de entrevista es una de la más utilizadas en la fenomenología, ya que proporciona a los participantes la oportunidad de poder extenderse en su experiencia con respecto al fenómeno de interés ${ }^{10}$. La pregunta que guió la entrevista fue: ¿Cuál ha sido su experiencia y sus necesidades al ser cuidador primario de un familiar con enfermedad de Alzheimer? La entrevista fue grabada y transcrita textualmente, palabra por palabra (verbatim).
Para el análisis de la información se utilizó el método de Colaizzi $(1978)^{10}$, que incluye las siguientes etapas: describir el fenómeno de interés, recolectar la descripción del fenómeno por los participantes, leer y releer todas las descripciones de los participantes sobre el fenómeno, volver a la transcripción original y extraer frases significativas frente a cada unidad de significado identificada, intentar explicar el significado de cada frase significativa, organizar los significados formulados en categorías más amplias, escribir una descripción exhaustiva del fenómeno develado y volver a los participantes para validar la descripción.

En relación con los aspectos éticos, se consideraron los principios éticos universales del Informe Belmont: respeto a las personas, beneficencia y justicia.

El principio de respeto a las personas plantea la convicción de que los individuos deben ser tratados como agentes autónomos. Según Acevedo, la autonomía se cumple cuando existe ausencia de control externo, intencionalidad y conocimiento ${ }^{11}$. En el presente estudio de caso se protegió este principio, aplicando un consentimiento informado previo a la entrevista, el cual proporcionó a la participante la información necesaria, por lo que pudo decidir de forma autónoma su participación. Además, se mantuvo la confidencialidad y el anonimato de la información entregada por la participante durante la entrevista: esta solo ha sido manejada por el investigador y se publicará únicamente en revistas científicas, manteniendo en reserva su nombre, por lo que en ningún caso podrá ser identificado. Debido a que la investigación cualitativa explora sobre la vivencia o experiencia de un fenómeno humano en particular, en ocasiones puede generar en el participante sensaciones dolorosas; en este caso, el investigador procurará el bienestar de la participante, ya sea poniendo fin a la entrevista u ofreciendo seguimiento, consejería o referencias. En esta entrevista la participante ha sido tratada con respeto y dignidad durante todo el proceso.

Para mantener el rigor científico de esta investigación se utilizaron los criterios de Guba $(1981)^{12}$ : credibilidad, confirmabilidad, frabilidad y tranferibilidad.

\section{- RESULTADOS}

El estudio incluyó una entrevista en profundidad en el mes de octubre de 2011, de 1 hora y 20 minutos de duración, a una mujer de 60 años, cuidadora primaria de un familiar con EA (concretamente su suegra) de 82 años de edad, con una progresión de la enfermedad de 5 a 6 años aproximadamente.

La entrevistada es viuda desde hace 30 años, tiene tres hijas, cada una de las cuales tiene su propia familia y habitan en sus propias domicilios, por lo que la cuidadora solo vive con su suegra y recibe la ayuda de una persona contratada para dicho fin.

Después de realizar la lectura repetitiva del verbatim y el análisis en profundidad de los relatos de la persona entrevistada, surgieron los siguientes significados en relación con la experiencia y las necesidades al ser cuidador primario de un familiar con EA.

\section{Experiencia fuerte debido al vínculo afectivo}

La persona entrevistada refiere que es una experiencia muy fuerte, cargada de sentimientos, lo cual se debe a la existencia de un vínculo afectivo con su suegra, una relación de amor y cariño, una relación muy estrecha y afianzada antes de la enfermedad, por lo que se hace muy difícil el hecho de aceptar la enfermedad:

"... Fuerte, fuerte, muy fuerte!!! es como fuerte realmente, cuando uno tiene un sentimiento con esta persona, como te digo cuando hay un sentimiento por medio, uno dice ¡cómo esta enfermedad tan desgraciada le vino a dar!...” 
Además, pone énfasis en la diferencia entre cuidar a una persona con la cual existe un vínculo previo, antes de la enfermedad, con conocerla ya con la enfermedad y cuidarla desde ese momento como ocurre con la persona que la ayuda con el cuidado de su suegra:

“... La chica que la cuida, la conoció así, en estas condiciones, entonces a ella no le afecta, ella no le afecta en nada..."

\section{Sentirse "violentada" y aparición de sentimientos contradictorios}

Este significado surgió repetitivamente durante la entrevista. La persona entrevistada refiere que se siente agredida y que además estas conductas siempre van dirigidas a ella. Por otra parte, está empezando a sentir miedo de estas conductas violentas. Toda esta situación le genera mucha angustia, debido a que no sabe cómo manejarla y enfrentarla:

"... Ella me agrede, y me agrede sabiendo que no soy yo y eso lo tengo claro..."

"...Sus reacciones conmigo son muy bruscas, muy violentas y por primera vez ayer a mi me dio susto, y dije yo, en una de estas agarran una fuerza terrible, porque si le he tenido que quitar cosas de sus manos y ella asi forcejeando y le busco por la buena y nada..."

"...Y justamente en un rato le dije mami deje ahi los cuchillos, yo los llevo y me hace asi (levanta la mano como amenazando) con la mano con el cuchillo y me dice yo los voy a llevar..."

Así también, el comportamiento de su suegra, que se encuentra enferma de EA, en ocasiones genera sentimientos encontrados o contradictorios, apareciendo sentimientos negativos como la rabia, la impotencia, el sentirse "manipulada", el cuestionarse si la enfermedad es real, si efectivamente ha perdido la memoria, si no recuerda a las personas. Pero al mismo tiempo se siente incómoda o culposa por pensar de esta manera:

“... Esas cosas son sentimientos encontrados y para luchar con eso cuesta, ¡esa impotencia!, jesa rabia!, ella no sabe no tiene idea lo que le pasa... esas respuestas asi tan altaneras eso descoloca demasiado, descoloca porque llega un momento que tú te quedas con la rabia de contestar y no poder contestar porque sabes que ella está enferma, tú te quedas con esa cosa adentro y eso al final te va haciendo daño..."

"... Pero cuesta asumirlo, uno lo tiene claro de aqui (se toca la cabeza) pero cuesta de acá (se toca el corazón), cuesta de acá, que te digan mil cosas y tú te quedes con eso adentro... eso es lo que duele, eso es lo que desgasta, porque que ellos sacan su rabia, su todo, pero uno se queda con la impotencia..."

"... Ya me decía y no me volvía a preguntar, entonces, yo pienso hasta qué grado, eso yo le digo a mi hija, a lo mejor yo estoy mal porque pienso que hasta qué grado de su enfermedad es verdad, o es manipulación, porque también hay mucha manipulación, no sé si lo hacen a sabiendas o lo hacen..."

\section{Sensación de pérdida del ser querido}

Esta unidad de significado surge debido a que el familiar no logra reconocer a su suegra en la persona que es actualmente, siente que es otra persona que no conoce y que actúa de una forma distinta a como actuaba anteriormente: al mirar a su suegra visualiza su cuerpo físico pero no su ser, es como si la esencia de la persona hubiese abandonado al cuerpo. Es como que el ser querido se va transformando en una persona extraña. Esto le produce mucha angustia, pero a la vez sabe que es ella y debe continuar cuidándola:
"... Ella no es la misma, ella ya no es, eso es lo triste, que no sea, eso es lo triste..."

".... Ya no me conoce, no, esta pérdida, o sea ya la perdimos..., ya la perdimos, entonces bueno, el tiempo que le queda que esté bien, que esté acogedora y con los remedios que uno pueda..."

"... Yo les digo a mis hijas, jla mami ya no está!, es feo decirlo y doloroso ¿no?, es como un bultito que se cae, es un bultito que hace tonteras porque no tiene mente, entonces es muy complicado, es muy complicada esta enfermedad..."

"... Ella fue una gran mujer, pero en estos momentos ella no tiene idea de nada, y era muy buena digo yo como, como una persona, por eso te digo, es muy complicado de verla en estas condiciones ahora... era una persona totalmente... diferente, una buena persona que le gustaba hacer el bien..."

"... una mujer no sé iquerendona! Verla ahora... yo sé que es ella, porque es su envase, ;pero lo de adentro no es ella!, y esa es como una lucha para uno..."

\section{Difícil decisión de institucionalización}

El desgaste que le provoca a la persona entrevistada asumir el cuidado de su suegra con EA lleva a cuestionarse la necesidad de internarla en un hogar de larga estancia. Esta no es una decisión fácil, debido al cariño y amor que se siente hacia la persona enferma, por todo lo vivido anteriormente. Además, existe un sentimiento de agradecimiento por el apoyo y carińo entregado, y una constante preocupación por asegurar una vida agradable hasta el momento de su muerte:

"... Lo hemos conversado mucho con mis hijas y hemos llegado asi que la vamos a internar, pero a mi se me parte el alma la verdad de las cosas, me parte el alma!!! porque yo sé que es ella, ella sabe que yo no soy, pero yo sí sé que es ella, que no es la misma persona, no es, pero es ella, ella la que nos entregó mucho cariño, es ella que nos ayudó mucho entonces..."

"... Pensamos internarla... una buena solución, pero a la vez yo digo cuánto tiempo más le quedará?, ¿cómo irá a ser si ella está internada? Ella tiene momentos de lucidez muy cortitos, pero los tiene, por ejemplo con una nieta mía tiene dos años entonces con ella, ella es otra, es la ternura, entonces una la mira, la observa y digo si la internamos eso ella va extrañar... entonces es tomar esas decisiones es como y para uno es muy fuerte..."

"... Entonces pienso que tengo que hacerlo hasta el final, ya estoy en esto, pero también pienso que yo también tengo que vivir, que también tengo que hacer mi vida, también tengo que hacer no sé, lo que yo quiera hacer, no voy a estar eternamente con ella ahi, porque yo voy a terminar peor..."

\section{Búsqueda del sentido de la enfermedad}

Como esta enfermedad es muy devastadora, ya que produce muchos cambios en el ámbito familiar, es necesario buscarle un sentido, para poder entender el porqué de esta enfermedad y cuál es la misión de que un familiar cercano padezca esta enfermedad.

“... Entonces a las finales qué piensas tú, esto es un castigo, estoy pagando algo, porque tú no estás viviendo bien tu vida, ¿qué es lo que estoy pagando?, ¿qué es lo que hice?...”

"... Nos mandan no más lo que tengamos que estar acá, y a veces digo y, algo tendrá que hacer más ella acá, alguna tarea, alguna misión, un algo en esas condiciones, a lo mejor... esta misma entrevista estaba para que... quién sabe, si uno se pone a pensar..."

\section{Sentirse sobrepasada por la situación}

La persona entrevistada se siente cansada, agotada y sobrepasada en algunas ocasiones por la situación de cuidado, pero este agotamiento afecta mucho más a la esfera psicológica que a la física, lo cual se ve reflejado 
en la aparición de cambios en la forma de responder al medio, con la aparición de conductas nuevas, negativas o agresivas. Además, al actuar de esta manera, en algunas ocasiones no se logra reconocer:

“... Lloré, lloré porque no habia llorado, me sentí como sobrepasada, entonces es cansador, es agotador, entonces yo esas cosas digo estoy 'colapsá..."

"... Yo sé que este problema como que me está agobiando mucho, entonces es cansador, es agotador, es muy agotador eso..."

"... Es muy desgastador, es muy desgastador, y psicológicamente porque, uno funciona, uno puede seguir andando, puede seguir haciendo cosas y todo, pero psicológicamente el cansancio es más terrible y con un enfermo de este tipo es muy desgastador..."

"...Yo siento de que a veces no tengo buenas reacciones conmigo misma (caen lágrimas), de contestar asi violento, contestar asi de mala forma, lo que yo no soy asi, no soy así..."

En relación con las necesidades al ser cuidador primario de un familiar con EA, surgieron las siguientes unidades de significados.

\section{Necesidad de información con respecto a la evolución de la enfermedad, tratamiento y sobre el cuidado}

Existe una necesidad de conocimiento en relación con la evolución de la enfermedad, su tratamiento y cuáles son los cuidados más adecuados para la persona enferma de EA, conocer qué ocurre en cada una de las etapas de la enfermedad y cuáles son los cuidados para cada una de ellas. Además, existe una necesidad de feedback continuo, en torno a si son correctos los cuidados que está entregando, que alguien la refuerce, guíe y le refiera cómo lo está haciendo.

“... Más conocimiento, más conocimiento de esta enfermedad porque no es mucho lo que se habla, no es mucho el conocimiento de que, o sea puede tener esta etapa, puede tener la otra etapa, la otra, la otra o la otra, pero qué pasa en esas etapas, cómo enfrentar esas etapas..."

"... ;Viendo la evolución como va!, cómo va eso, yo no sé si esta enfermedad será, irá a seguir peor o llegará un momento que se estanca iy eso a uno la tiene como en ascuas! sin saber, entonces es como vivir en ascuas, todos los dias..."

"... Puede reaccionar así, puede reaccionar asá, o sea tú tienes que saber que va a tener esas reacciones y que van ser por un tiempo, pero asi vivir que hasta cuándo va estar así, es como no saber, para mi es eso, hasta cuándo, por ejemplo el episodio de ayer, cuánto le va a durar..."

"... O te digan nooo te estás equivocando porque uno está haciendo las cosas pero uno no sabe si están bien hechas o no están bien hechas; para fortalecerse mentalmente, para dormir tranquila es que haya alguien atrás que te ayude..."

\section{Necesidad de apoyo psicológico}

La persona entrevistada menciona que considera necesario contar con apoyo psicológico por profesionales de área, ya sea tanto con la administración de medicamentos como con terapias psicológicas, que le permita contar con un apoyo y, además, alcanzar un descanso. También refiere una necesidad de poder relatar lo que le ocurre y que la "escuchen", lo que le permite desahogarse:

“... Yo creo de que la persona que esté así cuidando a un enfermo de eso tiene que tener unos apoyos muy grandes; por ejemplo, yo me he hecho asesorar por un psiquiatra, yo me estoy haciendo ver por un psiquiatra para que me eche una manita con alguna cosa, de estar más relajada, no sé, para poder dormir..."

"...Y como te digo yo, realmente las personas que cuidan este tipo de pacientes tienen que tener un apoyo, pero un apoyo de verdad, un apoyo de verdad, psicológico, un apoyo de verdad de amistad, un apoyo de no sé que, te saque de esto y te diga ;no si 'estay' bien!..."

"... Entonces para llegar al final juno tiene que estar bien! Y para eso yo encuentro de que ahora yo necesito estar bien y eso ya lo conversamos con las chiquillas, con mis hijas, le dije yo que me voy a ver un tratamiento porque la verdad es que necesito..."

"... Mira, asi como estamos conversando porque a veces uno necesita conversar este tipo de cosas así, porque a nadie por ejemplo, yo con mis redes no puedo conversar todo esto que estamos conversando las dos, porque ;se aburren! Se aburren que el tema sea el mismo y es totalmente, como decirte, es válido, porque no es un tema agradable, es un tema que todos le hacen el quite..."

\section{- DISCUSIÓN Y CONCLUSIÓN}

Se reveló el significado de la experiencia de ser cuidador primario de un familiar con EA y sus necesidades al entregar el cuidado, en una mujer de 60 años, chilena, que cuida de su suegra que padece esta enfermedad, a través de un estudio de tipo fenomenológico.

La primera unidad de significado denominada "experiencia fuerte debido al vínculo afectivo" puede relacionarse con lo citado por Esandi $\& \mathrm{Ganda}^{13}$, donde mencionan que una relación más cercana provoca niveles más altos de sobrecarga puesto que el componente afectivo desencadena una mayor implicación en el cuidado.

El "sentirse violentada" es otra unidad de significado que emergió del análisis en profundidad: la persona entrevistada se siente agredida y teme estas conductas agresivas. Una situación perturbadora para el bienestar del cuidador son las conductas de agresividad, entre las que se encuentran el rechazo, de manera verbal o física, a la ayuda o al cuidado que el cuidador otorga al paciente ${ }^{14}$. Así también, sienten terror de las reacciones, cada vez más violentas (primero insultos, luego golpes), lo que los agota y desvela ${ }^{15}$.

La "aparición de sentimientos contradictorios" en respuesta a los comportamientos de la persona enferma de EA genera en el familiar sentimientos negativos como la rabia, la impotencia, el sentirse "manipulada", pero al mismo tiempo emerge un sentimiento de culpabilidad al sentir lo mencionado anteriormente. En un estudio cualitativo realizado por Algado, Basterra y Garrigós, los autores observaron que los enfermos provocan en los cuidadores sentimientos contradictorios que muchas veces no pueden expresar abiertamente, ya sea por problemas de conciencia, escrúpulos o valores morales fuertemente interiorizados (16). Por un lado, sienten un gran carińo hacia el familiar enfermo, les produce muchísima tristeza verlos en esas condiciones, y sienten mucha pena y lástima, sobre todo cuando no pueden comunicarse con ellos o cuando ni siquiera les reconocen. Pero, por otro lado, los cuidadores tienen motivos para irritarse y exasperarse, lo que les lleva en ocasiones a sentir rabia hacia los enfermos y sobre todo hacia la situación en ocasiones conflictiva que crean.

"Sensación de pérdida del ser querido": esta unidad de significado surge debido a que el cuidador no logra reconocer a su familiar que presenta EA. Resultados similares fueron encontrados por Muñoz, Arancibia y Pare$\operatorname{des}^{15}$ : en su estudio cualitativo mencionan que el familiar cuidador describe cómo el enfermo se va transformando hasta ser un extrańo para la familia.

"Difícil decisión de institucionalización": en relación con esta unidad de significado que surgió en el presente estudio de caso, no se encontraron investigaciones que lo aborden en profundidad; se menciona que la decisión de la institucionalización de la persona enferma es un tema generador de conflicto dentro del entorno familiar ${ }^{13}$. Otros autores observaron que, a pesar de que el cuidado les produce cansancio y sentimientos de incapacidad con relación a la atención del paciente, algunos 
consideran que esta actividad no puede quedar en manos de personas extrañas, pues esta es responsabilidad de los hijos ${ }^{15}$.

El "sentirse sobrepasada por la situación" se relaciona con la gran carga que conlleva tener un familiar con EA; esto lleva a sentir agotamiento y de forma compensatoria a reaccionar de manera negativa, como por ejemplo contestar de forma violenta o inadecuada a otras personas de la familia. En un estudio cuantitativo realizado en Cuba se encontró que una de las dificultades más apremiantes identificadas en la vida diaria de los cuidadores primarios para ofrecer cuidados integrales a la persona con demencia fue el agotamiento físico y psicológico, que fue notificado por el $92 \%$ de los cuidadores ${ }^{7}$. Una visión cualitativa de este tema muestra que los efectos del cuidar más mencionados por los entrevistados son: agotamiento, estrés, intranquilidad, mal dormir y angustia. Además, asumir el cuidado impacta en el ocio y tiempo libre del cuidador primario, sus costumbres se ven alteradas, las vacaciones son casi inexistentes ${ }^{16}$.

En otro estudio cualitativo se halló que el cansancio experimentado por las cuidadoras no es solo físico, sino que se sienten solas y aisladas, que todos los días son iguales cuidando a personas con las cuales no pueden interactuar por su deterioro cognitivo, "un cansancio de no haber esperanza, un cansancio, en suma, encarnado en su hábitat y cotidianidad"17.

Por consiguiente, para poder manejar esta situación de agotamiento y cansancio, es necesario el descanso. En un estudio cualitativo realizado por De La Cuesta ${ }^{17}$ se observó que "descansar para una cuidadora es algo más que ser sustituida por unas horas, hacer algo por distraerse o salir a la calle, en definitiva es una experiencia lejana”; por lo tanto, el descanso se relaciona con el poder desconectarse del cuidado y para esto es necesario que las cuidadoras se sientan tranquilas con ellas mismas, con los otros y con la situación. Es decir, alcanzar la tranquilidad les permite desconectarse del cuidado y finalmente descansar.

Otra de las situaciones que permite aminorar esta situación de "sentirse sobrepasada" es contar con ayuda y apoyo para el cuidado, ya sea de amigos, familiares o profesionales; esto influye positivamente, puesto que tener un respiro y tiempo para dedicarse a uno mismo reduce el sentimiento de sobrecarga ${ }^{13}$.

Un alto nivel de apoyo social se relaciona con menor carga del cuidador, debido a que es una importante fuente de ayuda para los cuidadores en el momento de hacer frente a estas situaciones ${ }^{6}$. Pedir ayuda a algún familiar o a las personas que habitualmente les apoyan han sido las posibles soluciones para disfrutar de unos días de descanso ${ }^{16}$.

La "búsqueda del sentido de la enfermedad": esta unidad de significado queda expresada con la necesidad del familiar cuidador de encontrarle un significado a esta situación de enfermedad. El significado que los familiares atribuyen a la enfermedad son aspectos que influyen en el cuidado prestado a la persona enferma ${ }^{15}$.

En relación con las necesidades al ser cuidador primario de un familiar con EA, surgieron las siguientes dos unidades de significados: "necesidad de información con respecto a la evolución de la enfermedad, tratamiento y sobre el cuidado" y "necesidad de apoyo psicológico".

Algunos estudios refieren que entre las necesidades identificadas por las cuidadoras pueden mencionarse capacitación y mayor información sobre la enfermedad y sus nuevos hallazgos, su tratamiento y su evolución, y sobre los efectos en el entorno familiar ${ }^{7,16}$. De ahí la importancia para ellos de asistir a conferencias o cursos que contribuyan a otorgar un mejor cuidado y que los ayude a hacer frente a los cambios que la enfermedad lleva consigo y en definitiva a que les sirvan de apoyo moral y social, ${ }^{7,16}$.

Martínez ${ }^{7}$ encontró en su estudio el importante papel educativo de los profesionales de enfermería de la atención primaria de salud, como único enlace informativo para aprender sobre estos temas.

La aplicación de un programa de formación del cuidador en la demencia, realizado por un profesional de enfermería, pudo detectar un cambio en la reacción de los cuidadores ante los problemas conductuales y una mejoría en la afectividad de los pacientes. Es lógico pensar que la disminución del estrés del cuidador ante los trastornos del comportamiento sea un efecto directo de la intervención, que incluía la formación en el manejo de las alteraciones conductuales y en las técnicas de afrontamiento del estrés ${ }^{18}$.

Este estudio de caso de aproximación fenomenológica pretende ser una primera instancia que concederá a la enfermería la posibilidad de obtener un conocimiento más profundo y comprensivo en relación con la experiencia de ser cuidador primario de un familiar con Alzheimer y poder identificar sus necesidades, desde el contexto nacional. Así también, permite identificar las necesidades de una cuidadora de un familiar con EA, lo que proporciona algunos lineamientos en torno a la generación de programas de cuidado innovadores centrados en la particularidad de los usuarios, lo cual repercutirá en la calidad de vida de las personas que presentan la EA, los cuidadores y sus familias.

Para finalizar, es necesario mencionar que resulta imprescindible continuar profundizando en la investigación relacionada con el cuidado de las personas con EA y sus familias, debido a que cada día esta enfermedad presenta una mayor prevalencia derivada del aumento de la expectativa de vida a nivel mundial.

\section{- AGRADECIMIENTOS}

\section{A la Sra. María por compartir su experiencia}

\section{- BIBLIOGRAFÍA}

1. SENAMA [lnternet] Chile: Servicio Nacional del Adulto Mayor; c19982012. Propuesta de acción: Las personas mayores y los trastornos demenciales en Chile. [acceso 28 de marzo de 2012. Disponible en: http://www. senama.c//Archivos/Alzheimer/4.pdf

2. Badia X, Lara N, Roset M. Calidad de vida, tiempo de dedicación y carga percibida por el cuidador principal informal del enfermo de Alzheimer. Aten Primaria 2004: 34: 170-7.

3. Llibre J, Guerra M. Actualización sobre la enfermedad de Alzheimer. Rev Cubana Med Gen Integr [Internet]. 2002 [acceso 30 de octubre de 2012]; 18(4). Disponible en: http://scielo.sld.cu/scielo.php?script=sci_arttext\&pi$d=\$ 0864-21252002000400007$

4. Muela J, Torres C, Peláez E. Nuevo instrumento de evaluación de situaciones estresantes en cuidadores de enfermos de Alzheimer. Anales de Psicología 2002: 18: 319-31.

5. Pérez M. Las intervenciones dirigidas a los cuidadores de adultos mayores con enfermedad de Alzheimer. Revista Habanera de Ciencias Médicas [Internet]. 2008 [acceso 30 de octubre de 2012]; 7 (3). Disponible en:
http://redalyc.uaemex.mx/src/inicio/ArtPdfRed.jsp?iCve=180418872009 6. Molina J, lañez M, lañez B. El apoyo social como modulador de la carga del cuidador de enfermos de Alzheimer. Psicología y Salud 2005; 15: 33-43.

7. Martínez F. Necesidades de aprendizaje del cuidador principal para el tratamiento del anciano con demencia tipo Alzheimer. Rev Cubana Enferm [lnternet] 2009 [acceso 30 de octubre de 2012]; 25(3-4). Disponible en: http://scielo.sld.cu/scielo.php?pid=\$0864-03192009000200006\&scrip$\mathrm{t}=$ sci_arttex

8. Artasoa B, Goñib A, Biurrunc A. Cuidados informales en la demencia: predicción de sobrecarga en cuidadoras familiares. Rev Esp Geriatr Gerontol 2003; 38: 212-8.

9. Burns N, Grove S. Investigación en Enfermería. 3. ${ }^{\text {a }}$ ed. Madrid: Elsevier; 2004. pp. 554.

10. Streubert $\mathrm{H}$, Carpenter $\mathrm{D}$. Qualitative research in nursing: Advancing the humanistic imperative. 2. ${ }^{\text {a }}$ ed. Philadelphia: Lippincott; 1999.

11. Acevedo I. Aspectos éticos en la investigación científica. Ciencia y Enfermería 2002; 8: 15-8.
12. Streubert H, Carpenter D. Qualitative research in nursing: Advancing the humanistic imperative. 4. ${ }^{a}$ ed. Philadelphia: Lippincott; 2007.

13. Esandi N, Canga-Armayo A. Familia cuidadora y enfermedad de Alzheimer: una revisión bibliográfica. Gerokomos 2011:22: 56-61.

14. Arroyo E, Torres J, Castañeda JC. Agitación/agresividad en la demencia de tipo Alzheimer. Rev Esp Geriatr Gerontol 2001; 36:156-62.

15. Muñoz LA, Arancibia P, Paredes L. La experiencia de familiares cuidadores de pacientes que sufren Alzheimer y competencias del profesional de Enfermería. Invest Educ Enferm 1999; 17.

16. Algado MT, Basterra A, Garrigós I. Familia y enfermedad de Alzheimer. Una perspectiva cualitativa. Anales de Psicología 1997: 13: 19 -29.

17. De La Cuesta C. "Estar tranquila": Ia experiencia del descanso de cuidadoras de pacientes con demencia avanzada. Pensar Enfermagem. 2009; 13 : $2-9$

18. Sánchez P, Mouronte P, Olazarán J. Beneficios de un programa de formación del cuidador en la demencia: experiencia piloto desde la enfermería especializada. Rev Neurol 2001; 33: 422-24. 